\title{
THEREDA - Thermodynamic Reference Database
}

\author{
HELGE MOOG ${ }^{1}$, FRANK BOK ${ }^{2}$, DANIELA FREYER ${ }^{3}$, \\ XAVIER GAONA ${ }^{4}$ AND TRES THOENEN ${ }^{5}$ \\ ${ }^{1}$ Gesellschaft für Anlagen- und Reaktorsicherheit \\ ${ }^{2}$ Institute of Resource Ecology, Helmholtz-Zentrum Dresden- \\ Rossendorf \\ ${ }^{3}$ Technische Universität Bergakademie Freiberg \\ ${ }^{4}$ Karlsruhe Institute of Technology \\ ${ }^{5}$ Paul Scherrer Institute \\ Presenting Author: helge.moog@grs.de
}

Part of the process to ensure the safety of radioactive waste disposal is the predictive modeling of the solubility of all relevant toxic components in a complex aqueous solution. To ensure the reliability of thermodynamic equilibrium modeling as well as to facilitate the comparison of such calculations done by different institutions it is necessary to create a mutually accepted thermodynamic reference database. To meet this demand several institutions in Germany joined efforts and created THEREDA [1].

THEREDA is a suite of programs at the base of which resides a relational databank. Special emphasis is put on thermodynamic data along with suitable Pitzer coefficients which allow for the calculation of solubilities in high-saline solutions. Registered users may either download single thermodynamic data or readyto-use parameter files for the geochemical speciation codes PHREEQC, Geochemist's Workbench, CHEMAPP, or TOUGHREACT. Data can also be downloaded in a generic JSON-format to allow for the import into other codes. The database can be accessed via the world wide web: www.thereda.de

Prior to release, the released part of the database is subjected to many tests. Results are compared to results from earlier releases and among the different codes. This is to ensure that by additions of new and modification of existing data no adverse side effects on calculations are caused. Furthermore, our website offers an increasing number of examples for applications, including graphical representation, which can be filtered by components of the calculated system.

[1] H. C. Moog, F. Bok, C. M. Marquardt, V. Brendler (2015): Disposal of Nuclear Waste in Host Rock formations featuring high-saline solutions - Implementation of a Thermodynamic Reference Database (THEREDA). Appl. Geochem. (55) 72-84. http://dx.doi.org/10.1016/j.apgeochem.2014.12.016 